\title{
A NEW CURVE FITTING TECHNIQUE FOR ANALYSIS OF' FREQUENCY-DEPENDENT LOSSY TRANSMISSION LINES
}

\author{
Yuichi TANJI, Yoshifumi NISHIO and Akio USHIDA \\ Dept. of Electrical and Electronic Engineering, Tokushima University \\ 2-1 Minami-Josanjima, Tokushima 770, JAPAN \\ E-mail: tanji@ee.tokushima-u.ac.jp
}

\begin{abstract}
Analysis of frequency-dependent lossy transmission lines is very important for designing the high-speed VLSI, MCM and PCB. The frequency-dependent parameters are always obtained as tabulated data. In this paper, a new curve fitting technique of the tabulated data for the moment matching technique in interconnect analysis are presented. This method based on Chebyshev interpolation enhances the efficiency of the moment matching technique.
\end{abstract}

\section{INTRODUCTION}

The high-speed performance of microwave or digital circuit systems is limited by the interconnect effects rather than the switching speed of semiconductor devices. When the operating frequency is increase, the current density of conductor tends to be great around the surface of the conductor. Due to the high packing density, the interconnects such as VLSI, MCM and PCB are closely placed on each other, and the current density is also great at the near side between conductors. They are known as the skin effect and proximity effect [1], respectively, thus the interconnects of high-speed integrated circuits have frequency-dependent characteristics. The frequency-dependent parapeters are always obtained by any numerical procedure and as tabulated data in real frequency. Therefore, the analysis of frequencydependent lossy transmission lines with tabulated data is very important for accurate analysis VLSI's circuits, MCM and PCB.

For such analysis, FFT based algorithm is very accurate. However, this method is not useful from computational point of view, because the system to be analyzed contain very large number of transmissions lines, and FFT based algorithm needs large number of data points. The moment matching technique [3], [4] are efficient and accurate for the interconnect analysis. Recently, these methods are extended to the frequency-dependent case [5], [6]. Since the moment matching techniques are essentially Padé approximation of any Laplace functions, if any transfer function are described in power series of complex $s$, the moment matching technique can be applied to the analysis. Thus the key technique in reference [5], [6] is how the tabulated data in real frequency is described in power series of complex $s$, and the piecewise polynomial approximation in [6] and the least square approximation in [5] are used.

In this paper, we provide a new curve fitting technique for the moment matching scheme in interconnect analysis. The proposed method is based on Chebyshev interpolation technique. Chebyshev polynomial is considered as an almost minimax approximate polynomial. Hence the proposed method based on Chebyshev interpolation gives a good approximation than one by means of the least square fitting [5]. Moreover, since the proposed method does not require for any matrix operation, this method does not suffer from the singularity problem in the least square fitting [7]. The polynomial must be constructed as having real coefficients due to realistic impedance or admittance functions. The discrete orthogonal property of Chebyshev polynomial allows us to construct the continuous polynomial with real coefficients, different from the piecewise one in [6].

In the numerical examples, the proposed method gives reliable results to the tabulated data in real frequency.

\section{FREQUENCY-DEPENDENT LOSSY TRANSMISSION LINES}

The frequency-dependent transmission lines are described by the Telegrapher's equation in the Laplace-domain:

$$
\frac{d}{d x}\left[\begin{array}{c}
\mathbf{V}(s, x) \\
\mathbf{I}(s, x)
\end{array}\right]=\mathbf{D}(s)\left[\begin{array}{c}
\mathbf{V}(s, x) \\
\mathbf{I}(s, x)
\end{array}\right]
$$

where

$$
\begin{aligned}
& \mathbf{D}(s)=\left[\begin{array}{cc}
\mathbf{0} & -\mathbf{Z}(s) \\
-\mathbf{Y}(s) & \mathbf{0}
\end{array}\right] \\
& \mathbf{Z}(s)=\mathbf{R}(s)+s \mathbf{L}(s), \quad \mathbf{Y}(s)=\mathbf{G}(s)+s \mathbf{C}(s) .
\end{aligned}
$$

The parameters $\mathbf{R}(s), \mathbf{L}(s), \mathbf{C}(s), \mathbf{G}(s)$ are per unit length registance, inductance, capacitance, conductance matrices, respectively, and these matrices are arbitrary functions of complex $s$. Actually, these matrices are not given as a funtion of complex $s$, but also tabulated data to some points, $j \omega_{2}$ 's on the imaginary axis.

In this paper, our aim is how to apply the moment matching scheme [3], [4] for solving (1). If any transfer functions are described in power series of complex $s$, we can apply the moment matching scheme to the analysis, because the moment matching scheme is essentially Padé approximation. Hence, the input $(x=0)$-output $(x=l)$ relations of transmission lines are described in power series of complex $s$ is the key technique. Assuming that the parameter matrices $\mathbf{R}(s), \mathbf{L}(s), \mathbf{C}(s), \mathbf{G}(s)$ are power series of complex $s$, the matrix exponential method [5] [6] is very powerful technique to describe the input-output relation. Here we briefly modify the matrix exponential method to increase the efficiency. 
Applying the matrix exponential method, the input output relation of Eq. (1) is given by

$$
\begin{aligned}
{\left[\begin{array}{l}
\mathbf{V}(s, l) \\
\mathbf{I}(s, l)
\end{array}\right] } & =\exp (\mathbf{F}(s) l)\left[\begin{array}{c}
\mathbf{V}(s, 0) \\
\mathbf{I}(s, 0)
\end{array}\right] \\
& =\sum_{n=0}^{\infty} \frac{1}{n !}(\mathbf{F}(s) l)^{n}\left[\begin{array}{c}
\mathbf{V}(s, 0) \\
\mathbf{I}(s, 0)
\end{array}\right] \\
& =\sum_{n=0}^{\infty} \mathbf{T}_{n} s^{n}\left[\begin{array}{c}
\mathbf{V}(s, 0) \\
\mathbf{I}(s, 0)
\end{array}\right]
\end{aligned}
$$

In reference [3], the convergence of infinite series (2) is illustrated grately depending on the length $l$ of transmission lines. If the convergence is smooth, the transmission lines must be divided in some regin. (This implies that $\exp \left(\mathbf{F}(s) l_{1}\right)$ rapidly converges than $\exp \left(\mathbf{F}(s) l_{2}\right)$, if $l_{1}<l_{2}$.) In this case, the following relation is very useful in order to get the whole characteristics of transmission lines:

$$
\exp (\mathbf{F}(s) l)=\exp \left(\mathbf{F}(s) \frac{l}{2}\right) \cdot \exp \left(\mathbf{F}(s) \frac{l}{2}\right)
$$

However, dividing the transmission lines requires for more computational cost, because this means that some equations are added to the circuit equation. Alternatively, $\exp \left(-\mathbf{F}(s) \frac{l}{2}\right)$ is multiplied from left side of Eq. (2) instead of dividing, and we can get the following relations:

$\exp \left(-\mathbf{F}(s) \frac{l}{2}\right)\left[\begin{array}{c}\mathbf{V}(s, l) \\ \mathbf{I}(s, l)\end{array}\right]=\exp \left(\mathbf{F}(s) \frac{l}{2}\right)\left[\begin{array}{c}\mathbf{V}(s, 0) \\ \mathbf{I}(s, 0)\end{array}\right]$

The relation (4) represents continuity of the voltages and currents at the center point of the transmission lines, whereas the relation (2) gives a relation of the output variables to the input. This means that the relation (4) is more effective than (2), because the complexity depends on the length of the transmission lines.

Assuming $\mathbf{F}(s)$ as $M$ degree matrix polynomial of complex $s$, the coefficients of the matrix exponential (2) is obtained in recursive mannar [5], [6]:

$$
\mathbf{T}_{n}=\left\{\begin{array}{l}
\sum_{j=-1}^{\infty} \mathbf{T}_{0, j} \quad(n=0) \\
\sum_{j=\operatorname{int}\left(\frac{2-1}{M}\right)}^{\infty} \mathbf{T}_{i, j} \quad(n \neq 0)
\end{array}\right.
$$

where

$$
\begin{aligned}
\mathbf{T} i, j= & \frac{l}{j+1} \sum_{k=0}^{\min (i, M)} \mathbf{F}_{k} \mathbf{T}_{i-k, j-1} \\
& (i=0, \ldots, j M, j \neq 0) \\
\mathbf{T} i, j= & \frac{l}{j+1} \sum_{k=i-j M}^{M} \mathbf{F}_{k} \mathbf{T}_{i-k, j-1} \\
& (i=j M+1, \ldots,(j+1) M, j \neq 0) \\
\mathbf{T} i, 0= & \mathbf{F}_{i} l \quad(i=0, \ldots, M) \\
\mathbf{T} i, 0= & \mathbf{I} .
\end{aligned}
$$

$\exp (-\mathbf{F}(s) l)$ can be calculated by multiplying $\mathbf{T}_{i, j}$ by $(-1)^{j+1}$. Although the matrix exponential (2) congerves after 40-50 terms, $\exp (\mathbf{F}(s) l / 2)$ rapidly converges than $\exp (\mathbf{F}(s) l)$, because the length of transmission lines is half.

Interchanging the elements of (4), we can get the ports relation of the transmission lines as follows:

$$
\sum_{n=0}^{\infty} \mathbf{P}_{n} s^{n}\left[\begin{array}{c}
\mathbf{V}(s, 0) \\
\mathbf{V}(s, l)
\end{array}\right]+\sum_{n=0}^{\infty} \mathbf{Q}_{n} s^{n}\left[\begin{array}{c}
\mathbf{I}(s, 0) \\
\mathbf{I}(s, l)
\end{array}\right]=\mathbf{0}
$$

\section{CHEBYSHEV INTERPOLATION SCHEME OF FREQUENCY-DEPIENDENT PARAMETERS}

In the previous section, the matrix exponential method is applied to the moment generation of lossy transmission lines with frequency-dependent parameters. Here, it is assumed that the parameters given as tabulated data to some point on imaginary axis are able to write in power series of complex $s$. So, the procedure for making the power series from the tabulated data is provided in this section.

\subsection{Curve Fitting Algorithm}

Let $r(s), l(s), c(s), g(s)$ be $(\mathrm{i}, \mathrm{j})$ element of $\mathbf{R}(s), \mathbf{L}(s), \mathbf{C}(s)$, $\mathbf{G}(s)$, respectively, where these values are given as tabulated data to some points, $j \omega_{i}$ 's on the imaginary axis. The $(i, j)$ element $z(s)=r(s)+j s l(s)$ of the series impedance matrix and $y(s)=g(s)+j s c(s)$ of the parallel admittance matrix are determined so that they satisfies

$$
\begin{gathered}
z\left(j \omega_{2}\right) \approx \sum_{k=0}^{N} z_{k}\left(j \omega_{i}\right)^{i}, \quad y\left(j \omega_{i}\right) \approx \sum_{k=0}^{N} y_{k}\left(j \omega_{i}\right)^{i} \\
(i=0,1, \ldots, N)
\end{gathered}
$$

where $N$ is the number of the data. Moreover the coefficients $z_{k}$ and $y_{k}$ are assumed as real numbers, which is a reasonable assumption due to realistic impedance or admittance functions.

Let us consider $\mathrm{z}(\mathrm{s})$ only, and $\mathrm{y}(\mathrm{s})$ can be obtained by the same procedure. In reference [5], eliminating the lossless part of $l(s)$ is introduced in order to approximate accurately, namely, the lossless part $l(\infty)$ is separated from $l(j \omega)$ such as

$$
l^{\prime}(j \omega)=l(j \omega)-l(\infty)
$$

Then, $z^{\prime}\left(j \omega_{\imath}\right)=r\left(j \omega_{i}\right)+j \omega_{i} l^{\prime}\left(j \omega_{i}\right)$ is intepolated by the Chebyshev series. A transform $x=\omega / \omega_{m}$ is used to convert $\omega \in\left[0, \omega_{m}\right]$ into $x \in[0,1]$. Assuming $z^{\prime}(-j \omega)$ is complex conjugate to $z^{\prime}(j \omega)$, the interpolated polynomial is obtained by

$$
z^{\prime}\left(j \omega_{m} x\right)=\sum_{k=0}^{N-1} a_{k} T_{k}(x)
$$

where the symbol $\sum^{\prime}$ denotes the summation with the first component divided by 2 and $T_{k}(x)=\cos k \theta$. From the discrete orthogonal property of Chebyshev polynomial, $a_{k}(k=0,1 \ldots, N-1)$ are given as follows: 
if $\mathrm{N}$ is odd,

$$
a_{k}= \begin{cases}\frac{2}{N} \sum_{i=0}^{\frac{N-3}{2}}\left\{2 r\left(j \omega_{m} \cos \theta_{i}\right) \cos k \theta_{\imath}\right. & \left.+r(0) \cos \frac{k \pi}{2}\right\} \quad(\mathbf{k}: \text { even }) \\ j \frac{2}{N} \sum_{i=0}^{\frac{N-3}{2}} 2 \omega_{m} \cos \theta_{\imath} l\left(j \omega_{m} \cos \theta_{2}\right) \cos k \theta_{i} & (\mathrm{k}: \text { odd })\end{cases}
$$

if $\mathrm{N}$ is even,

$$
a_{k}=\left\{\begin{array}{l}
\frac{2}{N} \sum_{i=0}^{\frac{N}{2}-1} 2 r\left(j \omega_{m} \cos \theta_{i}\right) \cos k \theta_{i} \quad \text { (k: even) } \\
j \frac{2}{N} \sum_{i=0}^{\frac{N}{2}-1} 2 \omega_{m} \cos \theta_{i} l\left(j \omega_{m} \cos \theta_{i}\right) \cos k \theta_{i} \\
\quad \text { (k: odd) }
\end{array}\right.
$$

where $\theta_{\imath}=\frac{2 i+1}{N+2} \pi(i=0,1, \ldots, N-1)$ are the Chebyshev points. Note that if $\mathrm{N}$ is even number, the information at $s=0$ does not reflect the fitting curve. Hence, $N$ is prefer to be odd number.

The coefficients of $a_{k}$ are real part or imaginary part only, thus we can derive the power series of $j \omega_{m} x$ having real coefficients. First, $z^{\prime}\left(j \omega_{m} x\right)$ of (9) is converted into a power series with respect to $x$. Using the recurrence formula of Chebyshev polynomial,

$$
\left\{\begin{array}{l}
T_{0}(x)=1, \quad T_{1}(x)=1 \\
T_{k+1}(x)=2 x T_{k}(x)-T_{k-1}(x),
\end{array}\right.
$$

Chebyshev polynomial $T_{k}(i=2,3, \ldots)$ is obtained by

$$
\begin{aligned}
& T_{2}(x)=2 x^{2}-1 \\
& T_{3}(x)=4 x^{3}-3 x \\
& T_{4}(x)=8 x^{4}-8 x^{2}+1 \\
& T_{5}(x)=16 x^{5}-20 x^{3}+5 x \\
& T_{6}(x)=32 x^{6}-48 x^{4}+18 x^{2}-1,
\end{aligned}
$$

As a result, the finite Chebyshev series (9) is converted into a power series with $x$ :

$$
z^{\prime}\left(j \omega_{m} x\right)=\sum_{k=0}^{N-1} b_{k} x^{k}
$$

From (12), $T_{2 m}(x)$ and $T_{2 m+1}(x)$ are even and odd functions, respectively. Thus, $b_{2 m}$ and $b_{2 m+1}$ in (13) are respectively real and imaginary part only as $a_{2 m}$ and $a_{2 m+1}$ in (9). Consequently, $z^{\prime}\left(j \omega_{m} x\right)$ is expressed in power series of $j \omega_{m} x$ with real coefficients:

$$
z^{\prime}\left(j \omega_{m} x\right)=\sum_{k=0}^{N-1} z_{k}^{\prime}\left(j \omega_{m} x\right)^{k}
$$

Table 1. Coefficients of power series given by the proposed method.

\begin{tabular}{|c|c|c|c|}
\hline & value & & value \\
\hline \hline$c_{0}$ & 3.448 & $c_{6}$ & $-3.292 \times 10^{-8}$ \\
\hline$c_{1}$ & 4.670 & $c_{7}$ & $1.956 \times 10^{-10}$ \\
\hline$c_{2}$ & $-1.684 \times 10^{-2}$ & $c_{8}$ & $-1.601 \times 10^{-11}$ \\
\hline$c_{3}$ & $5.196 \times 10^{-4}$ & $c_{9}$ & $3.295 \times 10^{-14}$ \\
\hline$c_{4}$ & $-3.196 \times 10^{-7}$ & $c_{10}$ & $-2.917 \times 10^{-15}$ \\
\hline$c_{5}$ & $4.447 \times 10^{-7}$ & & \\
\hline
\end{tabular}

where

$$
z_{k}^{\prime}=\left\{\begin{array}{cc}
(-1)^{\frac{k}{2}} \frac{b_{k}}{\omega_{m}^{k}} & (\mathrm{k}: \text { even }) \\
(-1)^{\frac{k+1}{2}} \frac{j b_{k}}{\omega_{m}^{k}} & \text { (k: odd) }
\end{array}\right.
$$

From (8), (14), a element of the series impedance matrix of transmission lines is described by

$$
z(s)=z_{0}+s\left(z_{1}+l(\infty)\right)+\sum_{k=2}^{N-1} z_{k}^{\prime} s^{k} .
$$

where all coefficients of $s^{k}$ are real numbers.

\subsection{Shifted Coefficients of Power Series}

When the multi-point Padé approximation [3], [4] is used to getting dominant poles, the shifted moments, the coefficients of Taylor expansion at an arbitrary point $s_{k}$ is needed. Thus, in section II $F(s)$ in (2) and the matrix exponential must be a matrix polynomial of complex $\sigma=s-s_{k}$.

Let be $\mathbf{F}(s) M$ degree matrix polynomial as (2), then $\mathbf{F}(s)$ is convered into a matrix polynomail of complex $\sigma=s-s_{k}$ :

$$
\mathbf{F}(s) \equiv F(\sigma)=\sum_{m=0}^{M} \sum_{i=0}^{m}\left(\begin{array}{c}
m \\
i
\end{array}\right) \mathbf{F}_{m} s_{k}^{m-i} \sigma^{i} .
$$

\section{NUMERICAL EXAMPLES}

To show the efficiency of our method, the 3-conductors transmission lines provided by M. Celik are considered. The frequency-dependent parameters are listed as the tables II, III in reference [5]. Using the proposed method in Sect. 3, $(2,2)$ element of the series impedance matrix is given by the 10-degree power series of complex $s$ :

$$
z_{22}(s)=\sum_{i=0}^{10} c_{i} s^{i}
$$

where each coefficient is listed in Table 1.

For comparison, the values of $z_{22}$ in $s=j \omega$ and the tabulated data are shown in Fig. 1. In this figure, the frequencies range is from 0 to $7 \mathrm{GHz}$. The proposed method gives a reliable result. The time- and frequency-domain responses in the example [5] can be calculated by the matrix exponential method in Sect. 2 and complex frequency hopping [3]. The circuit includes two identical 3-conductor transmission lines, 4 resisters, 7 capacitors and 1 inductors. Transient responses to a pulse input (0.8 [ns] pulse width, $0.1[\mathrm{~ns}]$ rise 


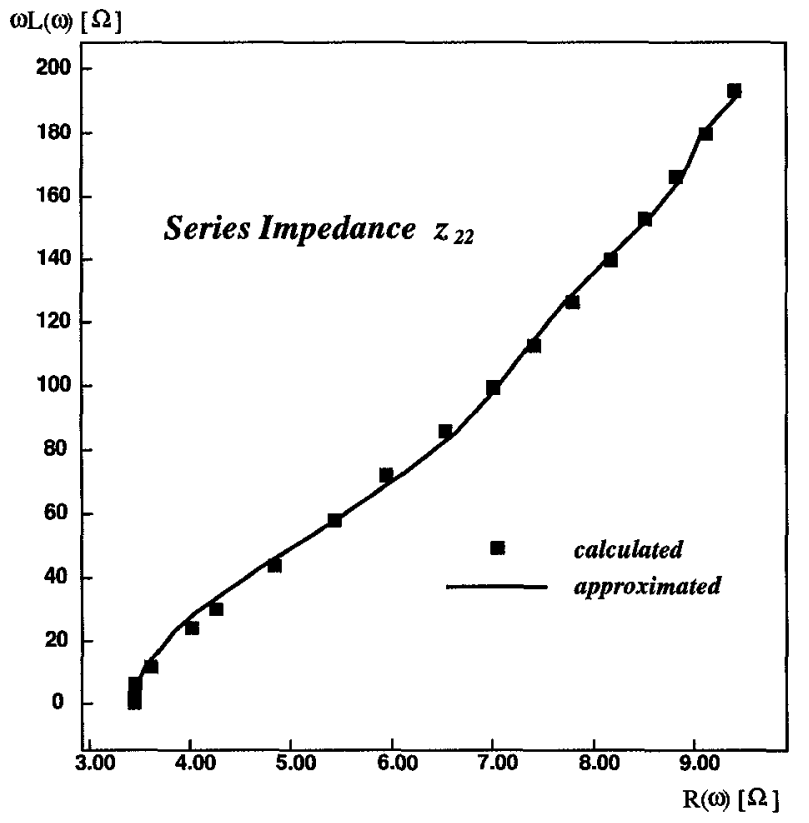

Figure 1. $(2,2)$ element of the series impedance matrix of the transmission lines provided by M. Celik [3].

and fall time) and frequency response to a impulse input are shown in Fig. 1, 2, respectively. These result are compared with the result by the frequency-domain method [8] and single Padé approximation [2]. Here, in complex frequency hopping, the maximum frequency is selected by 5 $[\mathrm{GHz}]$ and 9 expansion points is considered.

\section{CONCLUSIONS}

A new curve fitting technique for analysis of frequencydependent lossy transmission lines have been presented. This method is efficiently incorporated with the moment matching technique [3], [4]. Although the object of this paper is turned to the moment mathing technique, this method is easyly applied to the method of characteristics by means of a technique in reference [9]. This is our future work.

\section{REFERENCES}

[1] L. T. Hwang and I. Turlik, "A review of the skin effect as applied to thin film interconnects," IEEE Trans. Comp. Hybrids Manuf. Technol., vol. 15, vol. 15, no. 1, pp. 43-54, Feb. 1992.

[2] L. T. Pillage and R. A. Rohrer, "Asymptotic waveform evaluation for timing analysis," IEEE Trans. Computer-Aided Design, vol. 9, no. 4, April 1990.

[3] E. Chiprout and M. S. Nakhla, "Analysis of interconnect networks using complex frequency hopping (cfh)," IEEE Trans. Computer-Aided Design, vol. 14, no. 2, Feb. 1995.

[4] M. Celik, O. Ocali, M. A. Tan, and A. Atalar, "Improving awe accuracy using multipoint padé, approximation" Proc. ISCAS'94, vol. 1, pp. 379-382, 1994.

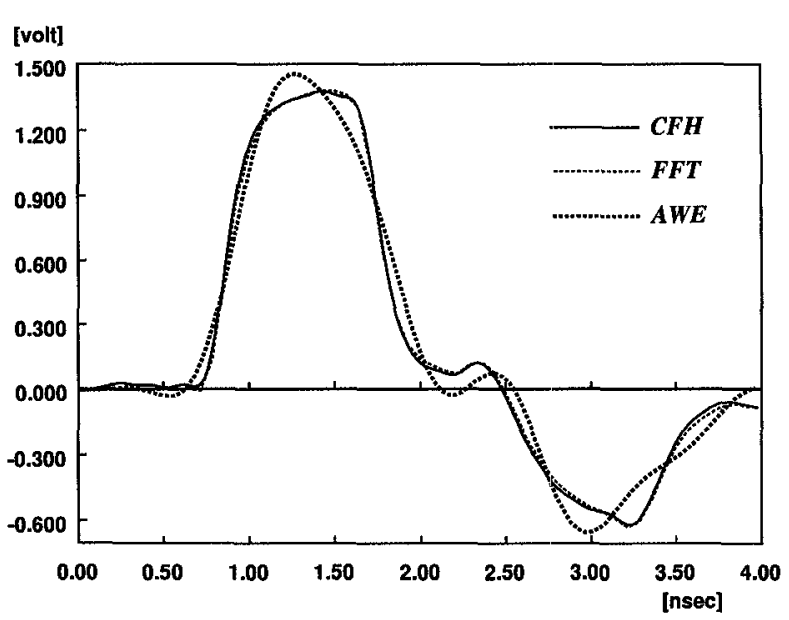

Figure 2. Transient response to a pulse input.

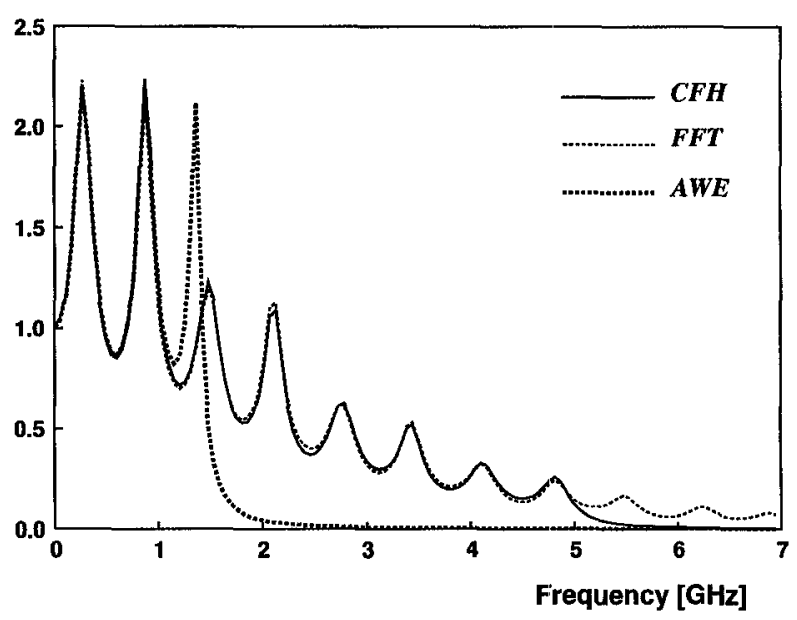

Figure 3. Frequency response to a impulse input.

[5] M. Celik and A. C. Cangellaris "Efficient transient simulation of lossy packaging interconnects using momentmatching techniques," IEEE Trans. Comps., Pack., \& Manuf. Technol., Part-B, vol. 19, no. 1, pp. 64-73, Feb. 1996.

[6] R. Khazaka, J. Poltz, M. Nakhla, Q. J. Zhang, "A fast method for the simulation of lossy interconnects with frequency dependent parameters," Proc. IEEE MultiChip Module Conf., pp. 95-98, Feb. 1996.

[7] G. H. Golub and C. F. Van Loan, Matrix Computation, the Johns Hopkins University Press, 1983.

[8] Y. Tanji, L. Jiang and A. Ushida, "Analysis of pulse responses of multi-conductor transmission lines by a partitioning technique," IEICE Trans. on Fundamentals, vol. E77-A, No. 12, pp. 2017-2027, Dec. 1994.

[9] T. Watanabe, A. Kamo and H. Asai, "Time-domain simulation of lossy coupled transmission lines based on delay evaluation technique," Proc. Euro. Conf. on Circuits Theory and Design, vol. 2, pp. 517-520, Sept. 1997. 